Regular article

\title{
Prediction of portal pressure from intra-operative ultrasonography
}

Atsushi Nanashima, MD, ${ }^{\mathrm{a}}$ Takafumi Abo, MD, ${ }^{\mathrm{a}}$ Junichi Arai, MD, ${ }^{\mathrm{a}}$

Tetsuro Tominaga, MD, ${ }^{\mathrm{a}}$ Katsunori Takagi, MD, ${ }^{\mathrm{a}}$ Koji Mochinaga, MD, ${ }^{\mathrm{a}}$

Katsuro Furukawa, MD, ${ }^{\mathrm{a}}$ Takeshi Nagayasu, $\mathrm{MD}^{\mathrm{a}}$

${ }^{a}$ Department of Surgical Oncology, Nagasaki University Graduate School of Biomedical Sciences, 1-7-1 Sakamoto, Nagasaki, Japan

Running title: Predicting portal pressure by ultrasonography

Corresponding author: Atsushi Nanashima, MD,

Department of Surgical Oncology, Nagasaki University Graduate School of Biomedical Sciences, 1-7-1 Sakamoto, Nagasaki 852-8501, JAPAN

Tel: +81-95-819-7304 Fax: +81-95-819-7306

E-mail: a-nanasm@nagasaki-u.ac.jp

This study was undertaken without financial support. No grants were received.

Nanashima is a main author who organizing entire study and write a paper. Nanashima will manage the total analysis, interpretation, data collection, writing the article, critical revision of the article. Abo, Arai, Tominaga, Takagi, Mochinaga and Furukawa are the co-operating colleagues who performed measuring portal pressure and hepatic flow velocity during operation. Abo and Nanashima performed statistical analysis. The present study is a prospective and consecutive study to clarify prediction of portal pressure by IOUS. 


\section{ABSTRACT}

Background: Portal hypertension is a major risk factor for hepatic failure or bleeding in patients who have undergone hepatectomy, but it cannot be measured indirectly. We attempted to evaluate the intra-operative ultrasonography parameters that correlate with portal pressure (PP) in patients undergoing hepatectomy.

Methods: We examined 30 patients in whom portal pressure was directly measured during surgery. The background liver conditions included chronic viral liver disease in seven patients, chemotherapy associated steatohepatitis in four patients, fatty liver in one patient, hepatolithiasis in one patient, obstructive jaundice in one patient and a normal liver in 16 patients. A multivariate logistic analysis and linear regression analysis were conducted to develop a predictive formula for PP.

Results: The mean PP was 10.4 $\pm 4.1 \mathrm{mmHg}$. The PP tended to be increased in patients with chronic viral hepatitis. A univariate analysis identified the association of the six following parameters with PP: the platelet count and the maximum (max), minimum (min), endo-diastolic, peak-systolic and mean velocity in the portal vein (PV) flow. Using multiple linear regression analysis, the predictive formula using the PV max and min was as follows: $\mathrm{Y}($ estimated PP $)=18.235-0.120 \mathrm{x}(\mathrm{PV} \max .(\mathrm{m} / \mathrm{s}))-0.364 \mathrm{x}(\mathrm{PV} \min )$. The calculated PP $(10.44 \pm 2.61 \mathrm{mmHg})$ was nearly the same as the actual PP $(10.43 \pm 4.07$ $\mathrm{mmHg}$ ). However, there was no significant relationship between the calculated PP and the intra-operative blood loss and posthepatectomy morbidity.

Conclusion: This formula, which uses ultrasonographic Doppler flow parameters, appears to be useful for predicting portal pressure.

Keywords: Hepatectomy, Intra-operative ultrasonography, Doppler-Portal pressure 


\section{Introduction}

The operative morbidity and mortality rates in patients who undergo hepatic resections have decreased in recent years due to improvements in both the preoperative evaluation of the liver's functional reserve and in perioperative management [1]. Portal hypertension remains a lethal operative risk in patients with liver dysfunction, such as cirrhosis, and should be carefully evaluated [2]. However, portal pressure (PP) can be measured directly by the insertion of a catheter via a trans-hepatic or trans-intestinal venous approach [3, 4]. This approach is an invasive and complicated measure, requiring either puncture of the liver or laparotomy. PP cannot currently be measured using indirect tests. In cases where evaluation is performed for pulmonary hypertension, the gradient flow velocity or the volume of the cardiac tricuspid valve on ultrasonography can be used to predict the pulmonary arterial pressure [5]. In addition, the gradient of the hepatic venous-portal pressure can be evaluated by various means [6-8]. However, indirectly evaluating PP itself remains difficult. Some investigators have attempted to identify the factors associated with portal hypertension [9-11], but clear predictors of PP have yet to be clarified.

Ultrasonography can be easily performed before or during surgery and can be used to measure the velocity, resistant index, etc. [12]. Intra-operative ultrasonography (IOUS) is an essential tool to determine tumor locations, surgical margins and adjacent vascular involvement. The accuracy of determining the flow Doppler by IOUS is high $(4,5)$. The flow Doppler's ultrasonographic parameters reflect the dynamics of the hepatic inflow and outflow, which may be associated with both the PP and the background liver status $[13,14]$. To the best of our knowledge, no indirect prediction of PP using ultrasonographic parameters has yet been reported. The analysis of PP using a predictive 
formula would be useful for evaluating operative risks and reducing portal hypertension-related hepatic complications in patients who undergo hepatectomy. We therefore attempted to examine the intra-operative ultrasonography parameters that correlate with PP in patients for whom PP could be measured directly during operation. We hypothesized that IOUS Doppler is a useful tool for evaluating hepatic vascularization and for accurately evaluating the correlation between the hepatic flow and the PP during surgery, which is important for evaluating portal hypertension.

The aim of the present preliminary study was to clarify aspects of IOUS that may predict portal pressure. For this purpose, as a preliminary study, the present study examined the relationships between the portal pressure and ultrasonographic parameters in 30 patients with various liver diseases in whom PP was directly measured to predict true PP. 


\section{Methods}

\subsection{Patients}

Subjects comprised 30 patients (21 men and nine women) with or without liver disease who underwent hepatectomies in the Department of Surgical Oncology at Nagasaki University Graduate School of Biomedical Sciences between February 2013 and January 2014. The 32 patients were consecutively entered for the present study and, however, two patients (metastatic liver carcinoma and hepatocellular carcinoma) could not technically underwent measuring the direct portal pressure because of the post-colectomy and post-cholecystectomy adhesions, respectively. The mean ( \pm standard deviation (SD)) age was $66 \pm 13$ years (range, 30 -85 years). The liver diseases warranting hepatic resection included hepatocellular carcinomas in 14 patients, metastatic liver carcinomas in eight patients, intrahepatic cholangiocarcinomas in three patients and bile duct carcinomas in five patients. The background liver condition was chronic viral liver disease in seven patients (including cirrhosis in two), chemotherapy associated steatohepatitis in four patients, fatty liver in one patient, hepatolithiasis in one patient, obstructive jaundice in one patient and normal livers in 16 patients.

In our hospital, the volume of the liver to be resected is estimated before surgery based on the results of the ICG retention rate at 15 min (ICGR15) using Takasaki's formula [15]. The liver volume, excluding the tumor, is measured by computed tomography (CT) volumetry [16]. Since 2004, we have modified our criteria and have used ${ }^{99 m}$ Tc-GSA scintigraphy and the associated functional hepatic volumetry, serum HA level and prothrombin activity based on ICGR15 testing to determine the extent of a hepatectomy [17]. In this cohort, we performed limited resections in five patients, a segmentectomy or sectionectomy in 12 patients, a lobectomy or more extended 
lobectomy in 10 patients and a pancreaticoduodenectomy in three patients. The study design was approved by the ethics review board at our university hospital, and informed consent for the data collection was obtained from each patient prior to participation.

\subsection{Evaluated parameters}

The clinical data, results of conventional liver function tests and surgical data were analyzed. The ICG was injected intravenously at a dose of $0.5 \mathrm{mg} / \mathrm{kg}$ body weight, and the 15-min retention rate was measured using a photopiece applied to the fingertip (RK-1000; Sumitomo Electric, Tokyo, Japan) without blood sampling [17, 18]. Patients received $3 \mathrm{mg}$ (185 MBq) of ${ }^{99 m}$ Tc-GSA (Nihon Medi-Physics, Nishinomiya, Japan) as a bolus dose into the antecubital vein. The clearance index of ${ }^{99 m}$ Tc-GSA (HH15) and the hepatic uptake ratio of ${ }^{99 m}$ Tc-GSA (LHL15; the count ratio in the liver compared with the sum of the count ratios for the heart and liver at 15 min after the injection of 99mTc-GSA) were calculated after the injection of ${ }^{99 m}$ Tc-GSA [18]. The HA was assayed using the sandwich binding protein assay (SRL, Tokyo, Japan). The normal value of the serum HA as determined by the laboratory data of SRL is $<50 \mathrm{ng} / \mathrm{ml}[135,17,18]$. Analysis of the histological fibrosis (staging) and necro-inflammatory responses (grading) were followed by calculating the Knodell score. [19].

\subsection{Direct measurement of portal pressure and the IOUS Doppler}

A 24-Fr venous catheter was inserted intra-operatively via the portal trunk, and the tip of the catheter was placed at the major portal trunk. Then, the catheter was connected with the anesthesia pressure monitoring system, and the PP was directly measured. The IOUS examination was performed using an XarioTM XG (Toshiba Medical Systems, 
Tokyo, Japan) and a micro-convex probe (PVT-375BT, 3.5 MHz, Toshiba) and an inter-operative probe (PLT-705BTH, 7.5 MHz). All IOUS were performed by surgeons to help with determining the indication for hepatectomy. First, we examined the two-dimensional (2D) images of the tumor and its location and also the Color Doppler Images of the tumor vasculature (12). For tumors located on the liver's surface, the liver was covered with warm saline to reduce the air-gap between the probe and the liver's surface. We separately scanned the hepatic flow in the portal vein and in the hepatic artery in the anterior segment of the liver and the middle hepatic vein before the hepatic transection. The parameters of the hepatic flow were the maximum velocity (max), the minimum (min) velocity, the endo-diastolic (ed) velocity, the peak-systolic (ps) velocity, the mean velocity, the pulsatility index (PI), the resistance index (RI) and the systolic/diastolic ratio (sd) of the portal vein and the hepatic artery (the secondary trunk of the anterior sector) and the trunk of the middle hepatic vein.

\subsection{Statistical analysis}

Continuous data are expressed as the mean \pm the SD. The data from the different groups were compared using a one-way analysis of variance and examined by Mann-Whitney's U-test. The comparison of the continuous data between groups was performed using Dunnet's multi-comparison analysis. The correlation between the continuous data was evaluated by a Pearson's correlation analysis, and the correlation coefficient (r) was calculated. A two-tailed p value $<0.05$ was considered to be statistically significant. The Statistical Package for the Social Science (SPSS) version 18.0 software (SPSS, Chicago, IL) was used for all statistical analyses. 


\section{Results}

\subsection{Clinicopathological parameters, portal pressure and US Doppler parameters}

Patient demographics and clinicopathological parameters were shown in Table 1. The preoperative liver function in the 30 patients was as follows: total bilirubin level, $0.82 \pm 0.39 \mathrm{mg} / \mathrm{dl}$; alanine aminotransferase, $30 \pm 18 \mathrm{IU} / \mathrm{l}$; platelet count, $18 \pm 6 \times 10^{4} / \mathrm{mm}^{3}$;

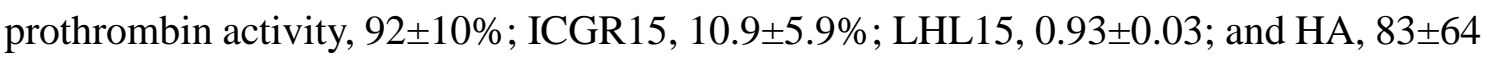
ng/ml. All patients showed Child-Pugh grades of A. The intra-operative blood loss was $806 \pm 711 \mathrm{ml}(15-2400 \mathrm{ml})$, and blood transfusions were performed in five patients. Postoperative complications were observed in two patients, including liver failure in one patient and an intra-abdominal infection in one patient. The staging of histological fibrosis was 0 in 19 patients, 1 in six patients, 2 in three patients and 4 in two patients. The grading of the histological necro-inflammatory responses was 0 in 18 patients, 1 in 10 patients and 2 in two patients.

IOUS Doppler liver scans were performed for all patients. No complications for measuring the direct portal pressure were registered in the present series. The portal pressure (PP) ranged between 4 and $18 \mathrm{mmHg}$, and the mean PP was $10.4 \pm 4.1 \mathrm{mmHg}$ (Table 1). The PP in the normal liver was $10.8 \pm 4.3 \mathrm{mmHg}, 8.3 \pm 3.4 \mathrm{mmHg}$ in chemotherapy-associated steatohepatitis, $8 \mathrm{mmHg}$ in fatty liver and obstructive jaundice, $7 \mathrm{mmHg}$ in hepatolithiasis and $11.8 \pm 4.5 \mathrm{mmHg}$ in chronic viral hepatitis, but there were no statistically significant differences between the groups. All of the US Doppler parameters of the 30 patients are summarized in Table 2.

3.2. Correlations between the liver functional parameters or the US Doppler parameters and the $P P$ 
Table 3 shows the correlations between the PP and the other comparable parameters. The correlation analysis identified five variables as significantly negatively correlated with PP: the PV max, PV min, PV ed, PV ps and the PV mean $(\mathrm{p}<0.05)$. The platelet counts tended to be negatively correlated with the PP but were not significant. Other parameters were not correlated with the PP in these 30 patients. Five US Doppler parameters were required for the multivariable analysis to predict PP before hepatectomy.

\subsection{Development of the predictive formula of portal pressure}

The five selected preoperative parameters were examined for correlations with PP using a multiple linear regression analysis with the stepwise method (Table 4). After four steps, the two parameters of PV max and PV min were still significant (see model 4 in Table 3). Based on this result, the regression equation was established as:

$\mathrm{Y}($ estimated PP $)=18.235-0.120 \times(\mathrm{PV}$ max. $)-0.364 \times(\mathrm{PV}$ min $)$

\subsection{Correlation between predictive portal pressure and actual portal pressure}

The mean estimated PP using the predictive formula was $10.44 \pm 2.61 \mathrm{mmHg}$ (range 3.9-14.3 mmHg), which was lower than the actual PP (10.43 $\pm 4.07 \mathrm{mmHg})$ (Table 1). The correlation between the actual PP and the estimated PP was statistically significant (correlation coefficient, 0.642; $\mathrm{p}<0.001$ ). The calculated PP was not significantly associated with the occurrence of postoperative complications (data not shown). A significant correlation between the blood loss and the calculated PP was not observed (correlation coefficient, 0.006; $\mathrm{p}=0.98$ ). 


\section{Discussion}

Portal hypertension is the most important consequence of cirrhosis, and its presence represents a hard endpoint for clinical outcomes such as varices, ascites, hepatorenal syndrome and encephalopathy [20]. The current standard for measuring portal hypertension and severity is usually the measurement of the hepatic venous pressure gradient. Measurement of this gradient is safe and relatively simple to perform, but it is invasive and costly $[6,7]$. Some of the surrogate hepatic markers correlating with PP have been examined because portal hypertension reflects hepatic dysfunctions [21, 22]. Zhang et al. and Wiechowska-Kozłowska et al. recently reported the usefulness of measuring the Doppler flow to evaluate portal hypertension $[23,24]$. Other investigators also examined the parameters of ultrasonic Doppler flow. However, distinct predictors representing PP have yet to be clarified.

The present study applied the reliable liver function markers of ICGR15, ${ }^{99 m}$ Tc-GSA liver scintigraphy and serum HA levels as well as conventional parameters $[13,14,17$, 18]. The ICGR15 and LHL15 from the ${ }^{99 \mathrm{~m}}$ Tc-GSA liver scintigraphy have been applied recently to evaluate the indications for surgical resection as a reliable marker $[14,17,18$, 25]. Our report showed a close correlation between the functional volumetry by ${ }^{99 m}$ Tc-GSA scintigraphy and PP [25], suggesting that HH15 and LHL15 are reliable predictors of PP. Serum HA is a marker of hepatic fibrosis and the deserialization of endothelial cell function in the liver [13], which reflect post-hepatectomy complications, such as hepatic failure $[13,26]$ that was caused by portal hypertension. However, in the small size of the present series, these parameters were not well correlated with PP. The platelet count tended to be negatively correlated with PP in the present study. The lower platelet counts reflected the severity of the hepatic damages related to high PP [27]. A 
previous study showed that platelet counts were markedly decreased according to the severity of portal hypertension [28].

By applying the US Doppler examinations, six parameters closely correlated with PP were identified. Among these parameters, the velocity of the portal flow was significantly associated with the PP value. In cases of portal hypertension, the velocity of the hepatic vein was expected to be decreased and flattened, and the arterial flow was expected to be increased [29]. However, in the present study, none of the hepatic artery and vein parameters were well correlated. Thus, portal velocity would be directly influenced by the PP. The location of the measurement may have an effect, although we measured the vessels in which the vertical flows could be easily observed. The portal flow velocities were negatively correlated with the PP in the present results, as indicated in the previous reports [29]. By applying these parameters, the prediction of PP would be possible. In selecting six parameters that could be evaluated preoperatively, the present study attempted to create a comprehensively predictive formula. To the best of our knowledge, an attempt to develop such a formula has not been fully reported. As a result of the multiple regression analysis, a formula for applying the two parameters of PV max. and PV min was developed but not for the mean velocity, RI or PI.

By comparing the calculated PP with the actual PP, a strong correlation could be clarified even though both the calculated PP and the actual PP were not always the same in each patient; thus, the calculated PP can be used to predict an estimated PP. To evaluate portal hypertension, the cut-off value should be established. As the definition of the upper limit of normal PP was $15 \mathrm{cmH}_{2} \mathrm{O}$ (approximately $11 \mathrm{mmHg}$ ), an estimated PP >11 mmHg was also thought to indicate an actual PP $>11 \mathrm{mmHg}$. An actual PP >11 mmHg was observed in 17 patients (57\%), whereas an estimated PP >11 mmHg was observed in 
14 patients. We believed that the calculated PP would be almost similar to the actual PP according to the present results. The relationship between the clinical outcomes and the calculated PP was considered to predict postoperative morbidity; however, hepatectomy-associated complications according to high PP were observed in only two patients in the present study. The correlation between the intra-operative blood loss and the calculated PP was not significant. To clarify the clinical significance of calculating the predictive PP from the US Doppler, a future study in a larger number of patients who have cirrhosis with portal hypertension or who had post-hepatectomy complications should be undertaken.

The usefulness of IOUS has been well established, and IOUS is considered a reliable imaging technique that is often used to confirm the diagnosis of intrahepatic tumor lesions [3, 4]. The advantages of IOUS Doppler were recently reported in patients undergoing hepatectomy [10-12]. In the present study, we assessed the usefulness of IOUS Doppler in the detection of portal hypertension in a preliminary study. Ideally, the measurement of the preoperative US would be useful to predict the operative risk of an increased PP. However, the extracorporeal US was often limited by the bowel gas or by the patients' physiques if they were obese. Based on this preliminary study, the usefulness of measuring the PV velocity with the IOUS Doppler to predict the PP was estimated; therefore, a further study with a larger number of patients will be necessary in the future. In recent years, hepatic elastography using a Fibroscan has been developed to detect liver injury or hepatic fibrosis [30]. The relationship between the PP and such a new diagnostic tool should be examined as well.

\section{Conclusion}


In conclusion, we have demonstrated that the IOUS Doppler allows us to evaluate the correlation with the portal pressure and to assist in making decisions about hepatic resections. Thirty patients underwent the measurement of PP during hepatic resections using intra-operative Doppler US parameters A predictive formula for PP using the portal flow velocity was determined, and the calculated values showed positive correlations with the true PP. As a relationship between the calculated PP and the postoperative outcomes or blood loss was not observed, a future study in a larger number of subjects using the present formula is needed. This imaging modality could become a useful surrogate procedure to evaluate portal hypertension in patients with various liver diseases who undergo hepatectomy.

Acknowledgements: None.

Conflict of Interest: None to declare. 


\section{REFERENCES}

(1).Poon RT, Fan ST, Lo CM, et al. Improving perioperative outcome expands the role of hepatectomy in management of benign and malignant hepatobiliary diseases: analysis of 1222 consecutive patients from a prospective database. Ann Surg. 2004;240:698-708

(2). Cucchetti A, Ercolani G, Vivarelli M, et al. Is portal hypertension a contraindication to hepatic resection? Ann Surg. 2009;250:922-8.

(3). Gibo M, Unten S, Yogi A, et al. Percutaneous ipsilateral portal vein embolization using a modified four-lumen balloon catheter with fibrin glue: initial clinical experience. Radiat Med. 2007;25:164-72.

(4). Shimura T, Suehiro T, Suzuki H, Okada K, Araki K, Kuwano H. Trans-ileocecal portal vein embolization as a preoperative treatment for right trisegmentectomy with caudate lobectomy. J Surg Oncol. 2007;96:438-41.

(5). Ling LF, Marwick TH. Echocardiographic assessment of right ventricular function: how to account for tricuspid regurgitation and pulmonary hypertension. JACC Cardiovasc Imaging. 2012;5:747-53.

(6). Chelliah ST, Keshava SN, Moses V, Surendrababu NR, Zachariah UG, Eapen C. Measurement of hepatic venous pressure gradient revisited: Catheter wedge vs balloon wedge techniques. Indian J Radiol Imaging. 2011;21:291-3

(7). Thalheimer U, Leandro G, Samonakis DN, Triantos CK, Patch D, Burroughs AK. Assessment of the agreement between wedge hepatic vein pressure and portal vein pressure in cirrhotic patients. Dig Liver Dis. 2005;37:601-8.

(8). Maleux G, Willems E, Fieuws S, et al. Prospective study comparing different indirect methods to measure portal pressure. J Vasc Interv Radiol. 2011;22:1553-8 
(9).Min YW, Bae SY, Gwak GY, et al. A clinical predictor of varices and portal hypertensive gastropathy in patients with chronic liver disease. Clin Mol Hepatol. 2012;18:178-84.

(10). Alempijevic T, Sokic-Milutinovic A, Milicic B, et al. Noninvasive assessment of portal hypertension in patients with alcoholic cirrhosis. Turk J Gastroenterol. 2012;23:239-46.

(11). Hayashi H, Beppu T, Okabe H, et al. Combined measurements of serum bile acid level and splenic volume may be useful to noninvasively assess portal venous pressure. J Gastroenterol. 2012;47:1336-41.

(12). Schneider PD. Preoperative assessment of liver function. Surg Clin North Am. 2004;84:355-73.

(13). Yachida S, Wakabayashi H, Kokudo Y, et al. Measurement of serum hyaluronate as a predictor of human liver failure after major hepatectomy. World J Surg. 2000;24:359-364.

(14). Mitsumori A, Nagaya I, Kimoto S, et al. Preoperative evaluation of hepatic functional reserve following hepatectomy by technetium-99m galactosyl human serum albumin liver scintigraphy and computed tomography. Eur. J Nucl. Med. 1998;25:1377-1382.

(15). Takasaki T, Kobayashi S, Suzuki S, et al. Predetermining postoperative hepatic function for hepatectomies. Int. Surg. 1980;65:309-313

(16). Kubota K, Makuuchi M, Kusaka K, et al. Measurement of liver volume and hepatic functional reserve as a guide to decision-making in resection surgery for hepatic tumors. Hepatology 1997;26:1176-1181. 
(17). Nanashima A, Tobinaga S, Abo $T$, et al. Reducing the incidence of post-hepatectomy hepatic complications by preoperatively applying parameters predictive of liver function. J Hepatobiliary Pancreat Sci. 2010;17:871-8.

(18) Nanashima A, Yamaguchi H, Shibasaki S, et al. Relationship between CT volumetry and functional liver volume using technetium-99m galactosyl serum albumin scintigraphy in patients undergoing preoperative portal vein embolization before major hepatectomy: a preliminary study. Dig Dis Sci. 2006;51:1190-5.

(19) Knodell RG, Ishak KG, Black WC, et al. Formulation and application of a numerical scoring system for assessing histological activity in asymptomatic chronic active hepatitis. Hepatology. 1981;1:431-435.

(20) Al-Busafi SA, McNabb-Baltar J, Farag A, Hilzenrat N. Clinical manifestations of portal hypertension. Int J Hepatol. 2012;2012:203794.

(21) Lim LG, Lee YM, Tan L, Chang S, Lim SG. Percutaneous paraumbilical embolization as an unconventional and successful treatment for bleeding jejunal varices. World J Gastroenterol. 2009;15:3823-6.

(22) Shneider BL, Abel B, Haber B, et al. Childhood Liver Disease Research and Education Network. Portal hypertension in children and young adults with biliary atresia. J Pediatr Gastroenterol Nutr. 2012;55:567-73.

(23) Zhang L, Yin J, Duan Y, Yang Y, Yuan L, Cao T. Assessment of intrahepatic blood flow by Doppler ultrasonography: relationship between the hepatic vein, portal vein, hepatic artery and portal pressure measured intraoperatively in patients with portal hypertension. BMC Gastroenterol. 2011;11:84. 
(24) Wiechowska-Kozlowska A, Raszeja-Wyszomirska J, Wasilewicz MP, et al. Upper gastrointestinal endosonography in patients evaluated for liver transplantation.Transplant Proc. 2009;41:3082-4.

(25) Nanashima A, Tobinaga S, Abo T, et al. Relationship of hepatic functional parameters with changes of functional liver volume using technetium-99m galactosyl serum albumin scintigraphy in patients undergoing preoperative portal vein embolization: a follow-up report. J Surg Res. 2010;164:e235-42.

(26) Mizuguchi T, Katsuramaki T, Nobuoka T, et al. Serum hyaluronate level for predicting subclinical liver dysfunction after hepatectomy. World J Surg. 2004;28:971-6.

(27) Ikeda N, Imanishi H, Aizawa N, Tanaka H, Iwata Y, Enomoto H, Saito M, Iijima H, Iimuro Y, Fujimoto J, Yamamoto S, Hirota S, Kudo M, Arii S, Nishiguchi S. Nationwide survey in Japan regarding splenectomy/partial splenic embolization for interferon treatment targeting hepatitis $\mathrm{C}$ virus-related chronic liver disease in patients with low platelet count. Hepatol Res. 2013. doi: 10.1111/hepr.12184.

(28) Alempijevic T, Sokic-Milutinovic A, Milicic B, Jesic R, Balovic A, Popovic D, Krstic M. Noninvasive assessment of portal hypertension in patients with alcoholic cirrhosis. Turk J Gastroenterol. 2012;23:239-46.

(29) Zhang L, Yin J, Duan Y, Yang Y, Yuan L, Cao T. Assessment of intrahepatic blood flow by Doppler ultrasonography: relationship between the hepatic vein, portal vein, hepatic artery and portal pressure measured intraoperatively in patients with portal hypertension. BMC Gastroenterol. 2011;11:84. 
(30) Sporea I, Raţiu I, Bota S, Şirli R, Jurchiş A. Are different cut-off values of liver stiffness assessed by transient elastography according to the etiology of liver cirrhosis for predicting significant esophageal varices? Med Ultrason. 2013;15:111-5. 
Table 1 Patient background, clinicopathological data and the measured portal pressures

\begin{tabular}{|c|c|c|c|c|c|c|c|c|c|c|c|c|c|c|c|c|c|}
\hline & Age & Gender & Disease & $\begin{array}{l}\text { Background } \\
\text { Liver }\end{array}$ & $\begin{array}{l}\text { Blood } \\
\text { Loss(ml) }\end{array}$ & Outcome & $\begin{array}{l}\text { ICGR15 } \\
\text { (\%) }\end{array}$ & LHL15 & $\begin{array}{l}\text { Total } \\
\text { bilirubin } \\
\text { (mg/dl) }\end{array}$ & $\begin{array}{l}\text { Alanine } \\
\text { transaminase } \\
(\mathrm{IU} / \mathrm{l})\end{array}$ & $\begin{array}{l}\text { Platelet } \\
\text { count } \\
\left(\mathrm{x} 10^{4} / \mathrm{mm}^{3}\right)\end{array}$ & $\begin{array}{l}\text { Prothrombin } \\
\text { activity (\%) }\end{array}$ & $\begin{array}{l}\text { Hyaluronic } \\
\text { Acid (ng/ml) }\end{array}$ & Staging & Grading & $\begin{array}{l}\text { Actual PV } \\
\text { pressure } \\
(\mathrm{mmHg})\end{array}$ & $\begin{array}{l}\text { Calculated PV } \\
\text { pressure } \\
(\mathrm{mmHg})\end{array}$ \\
\hline 1 & 54 & $\mathrm{f}$ & CCC & normal & 1080 & $\mathrm{n}$ & 15.0 & 0.93 & 1.1 & 20 & 13 & 87 & 95 & 0 & 0 & 17 & 14.12 \\
\hline 2 & 69 & $\mathrm{~m}$ & HCC & normal & 740 & $\mathrm{n}$ & 8.6 & 0.88 & 0.4 & 42 & 26 & 97 & 45 & 2 & 1 & 4 & 7.22 \\
\hline 3 & 30 & $\mathrm{~m}$ & MLC & normal & 1400 & $\mathrm{n}$ & 1.2 & 0.98 & 0.7 & 60 & 16 & 77 & 23 & 0 & 0 & 18 & 11.23 \\
\hline 4 & 64 & $\mathrm{f}$ & BDC & normal & 860 & $\mathrm{n}$ & 8.9 & 0.94 & 0.5 & 19 & 16 & 111 & 43 & 0 & 0 & 9 & 6.32 \\
\hline 5 & 65 & $\mathrm{~m}$ & BDC & jaundice & 850 & LF & 10.9 & 0.93 & 2.1 & 52 & 22 & 102 & 48 & 0 & 0 & 8 & 6.26 \\
\hline 6 & 36 & $\mathrm{f}$ & HCC & normal & 150 & $\mathrm{n}$ & 5.0 & 0.95 & 1.5 & 12 & 27 & 75 & 67 & 0 & 0 & 5 & 7.17 \\
\hline 7 & 72 & $\mathrm{~m}$ & MLC & CASH & 180 & $\mathrm{n}$ & 15.3 & 0.93 & 1.4 & 10 & 12 & 78 & 151 & 0 & 0 & 5 & 8.56 \\
\hline 8 & 80 & $\mathrm{~m}$ & MLC & CASH & 480 & $\mathrm{n}$ & 9.7 & 0.96 & 0.3 & 30 & 6 & 90 & 67 & 0 & 0 & 8 & 9.68 \\
\hline 9 & 85 & $\mathrm{f}$ & MLC & normal & 430 & $\mathrm{n}$ & 7.1 & 0.86 & 0.7 & 15 & 25 & 96 & 84 & 0 & 0 & 12 & 13.03 \\
\hline 10 & 67 & $\mathrm{~m}$ & HCC & normal & 1700 & $\mathrm{n}$ & 12.5 & 0.90 & 0.6 & 40 & 13 & 93 & 268 & 1 & 1 & 12 & 9.35 \\
\hline 11 & 65 & $\mathrm{f}$ & BDC & normal & 1450 & infection & 6.5 & 0.94 & 0.8 & 14 & 26 & 89 & 23 & 0 & 0 & 6 & 10.80 \\
\hline 12 & 63 & $\mathrm{f}$ & HCC & $\mathrm{CVH}$ & 480 & $\mathrm{n}$ & 14.0 & 0.93 & 0.7 & 46 & 20 & 105 & 107 & 1 & 1 & 10 & 12.32 \\
\hline 13 & 67 & $\mathrm{~m}$ & HCC & $\mathrm{CVH}$ & 870 & $\mathrm{n}$ & 13.5 & 0.92 & 1.1 & 16 & 17 & 88 & 110 & 4 & 1 & 14 & 14.29 \\
\hline 14 & 58 & $\mathrm{~m}$ & HCC & CVH(cirrhosis) & 2400 & $\mathrm{n}$ & 26.8 & 0.88 & 1.1 & 35 & 7 & 82 & 189 & 4 & 2 & 14 & 13.59 \\
\hline 15 & 84 & $\mathrm{f}$ & MLC & normal & 200 & $\mathrm{n}$ & 9.0 & 0.92 & 0.5 & 12 & 14 & 88 & 52 & 0 & 0 & 5 & 8.54 \\
\hline 16 & 64 & $\mathrm{f}$ & MLC & normal & 350 & $\mathrm{n}$ & 9.0 & 0.94 & 0.8 & 23 & 15 & 95 & 45 & 0 & 0 & 16 & 12.28 \\
\hline 17 & 76 & $\mathrm{~m}$ & HCC & CVH & 820 & $\mathrm{n}$ & 10.2 & 0.96 & 0.9 & 49 & 14 & 88 & 60 & 2 & 1 & 10 & 7.73 \\
\hline 18 & 56 & $\mathrm{~m}$ & BDC & normal & 920 & $\mathrm{n}$ & 10.5 & 0.95 & 1.3 & 85 & 19 & 86 & 78 & 0 & 0 & 8 & 11.04 \\
\hline
\end{tabular}




\begin{tabular}{|c|c|c|c|c|c|c|c|c|c|c|c|c|c|c|c|c|c|}
\hline 19 & 68 & $\mathrm{~m}$ & HCC & normal & 180 & $\mathrm{n}$ & 6.6 & 0.93 & 0.6 & 27 & 20 & 84 & 56 & 0 & 1 & 12 & 12.55 \\
\hline 20 & 82 & $\mathrm{~m}$ & HCC & normal & 1930 & $\mathrm{n}$ & 18.1 & 0.95 & 0.7 & 22 & 14 & 105 & 111 & 1 & 1 & 13 & 9.63 \\
\hline 21 & 62 & $\mathrm{~m}$ & HCC & CVH(cirrhosis) & 2030 & $\mathrm{n}$ & 13.8 & 0.94 & 0.5 & 26 & 18 & 93 & 101 & 1 & 1 & 14 & 12.61 \\
\hline 22 & 85 & $\mathrm{~m}$ & HCC & fatty & 15 & $\mathrm{n}$ & 11.0 & 0.96 & 0.6 & 15 & 27 & 76 & 97 & 1 & 2 & 8 & 9.72 \\
\hline 23 & 74 & $\mathrm{~m}$ & MLC & CASH & 850 & $\mathrm{n}$ & 7.0 & 0.94 & 0.3 & 18 & 19 & 92 & 115 & 0 & 0 & 13 & 13.30 \\
\hline 24 & 67 & $\mathrm{~m}$ & HCC & $\mathrm{CVH}$ & 2000 & $\mathrm{n}$ & 18.8 & 0.94 & 1.1 & 59 & 13 & 100 & 26 & 2 & 1 & 5 & 3.94 \\
\hline 25 & 72 & $\mathrm{~m}$ & HCC & normal & 230 & $\mathrm{n}$ & 8.9 & 0.93 & 1.0 & 15 & 17 & 96 & 45 & 1 & 1 & 12 & 12.45 \\
\hline 26 & 74 & $\mathrm{~m}$ & CCC & normal & 50 & Infection & 3.4 & 0.94 & 0.8 & 27 & 20 & 112 & 16 & 0 & 0 & 11 & 9.94 \\
\hline 27 & 58 & $\mathrm{f}$ & BDC & hepatolithiasis & 890 & $\mathrm{n}$ & 2.5 & 0.95 & 0.7 & 36 & 29 & 114 & 23 & 0 & 0 & 7 & 10.20 \\
\hline 28 & 59 & $\mathrm{~m}$ & CCC & normal & 750 & $\mathrm{n}$ & 4.0 & 0.93 & 0.6 & 16 & 14 & 88 & 98 & 0 & 0 & 12 & 12.98 \\
\hline 29 & 40 & $\mathrm{~m}$ & MLC & CASH & 700 & $\mathrm{n}$ & 9.8 & 0.95 & 0.4 & 23 & 19 & 87 & 45 & 0 & 0 & 7 & 11.83 \\
\hline 30 & 68 & $\mathrm{~m}$ & HCC & $\mathrm{CVH}$ & 110 & $\mathrm{n}$ & 21.4 & 0.89 & 0.8 & 44 & 18 & 89 & 154 & 0 & 0 & 18 & 10.46 \\
\hline
\end{tabular}

ICG: indocyanine green, LHL15: liver uptake ratio at 15 minutes

CCC: cholangiocellular carcinoma, HCC: hepatocellular carcinoma, MLC: metastatic liver carcinoma, BDC: bile duct carcinoma, CVH: chronic viral hepatitis, CASH: chemotherapy-associated steatohepatitis, LF: liver failure 
Table 2 Each parameter of ultrasonographic Doppler

\begin{tabular}{|l|l|l|l|}
\hline & Portal vein & Hepatic artery & Hepatic vein \\
\hline Maximum (m/sec.) & $28.4 \pm 11.5$ & $52.6 \pm 21.2$ & $-16.2 \pm 25.0$ \\
\hline Minimum (m/sec.) & $12.1 \pm 4.9$ & $17.9 \pm 9.0$ & $-4.2 \pm 12.5$ \\
\hline Endo-diastolic (m/sec) & $15.6 \pm 6.1$ & $22.2 \pm 11.8$ & $-5.9 \pm 10.9$ \\
\hline Peak-systolic (m/sec.) & $16.4 \pm 6.6$ & $32.7 \pm 15.9$ & $-7.7 \pm 10.9$ \\
\hline Mean (m/sec.) & $7.5 \pm 3.6$ & $14.8 \pm 6.9$ & $-4.0 \pm 4.7$ \\
\hline Pulsatility index & $0.84 \pm 0.66$ & $0.96 \pm 0.47$ & $1.92 \pm 1.33$ \\
\hline Resistance index & $0.47 \pm 0.28$ & $0.61 \pm 0.31$ & $0.74 \pm 0.34$ \\
\hline Systolic/diastolic ratio & $2.09 \pm 0.84$ & $2.94 \pm 2.47$ & $4.30 \pm 9.70$ \\
\hline
\end{tabular}


Table 3 Correlations between portal pressure and clinicopathological or liver functional parameters and parameters of ultrasonographic Doppler test.

\begin{tabular}{lcc}
\hline & $\begin{array}{c}\text { Correlation } \\
\text { (r value) }\end{array}$ & $\mathrm{P}$ \\
\hline ICGR15 (\%) & 0.162 & 0.421 \\
LHL15 & -0.058 & 0.776 \\
Total bilirubin (mg/dl) & -0.109 & 0.566 \\
Alanine transaminase (IU/L) & 0.031 & 0.872 \\
Platelet count (/mm ${ }^{3}$ ) & -0.314 & 0.091 \\
Prothrombin activity (\%) & -0.071 & 0.711 \\
Hyaluronic acid (ng/ml) & 0.251 & 0.273 \\
PV maximum (max.) & -0.496 & 0.005 \\
PV minimum (min.) & -0.558 & 0.001 \\
PV endo-diastolic (ed.) & -0.514 & 0.004 \\
PV peak-systolic (ps.) & -0.536 & 0.002 \\
PV mean & -0.430 & 0.018 \\
PV pulsatility index (PI) & 0.176 & 0.466 \\
PV resistance index (RI) & 0.126 & 0.515 \\
\hline
\end{tabular}




\begin{tabular}{lcc}
\hline PV systolic/diastolic ratio (s/d) & 0.164 & 0.396 \\
HA max. & 0.079 & 0.679 \\
HA min. & -0.126 & 0.506 \\
HA ed. & -0.196 & 0.301 \\
HA ps. & 0.078 & 0.682 \\
HA mean & 0.067 & 0.723 \\
HA PI. & -0.030 & 0.881 \\
HA RI. & -0.107 & 0.588 \\
HA s/d & 0.160 & 0.418 \\
HV max. & 0.129 & 0.496 \\
HV min. & 0.166 & 0.381 \\
HV ed. & 0.125 & 0.512 \\
HV ps. & 0.163 & 0.389 \\
HV mean & 0.154 & 0.426 \\
HV PI. & -0.124 & 0.513 \\
HV RI. & -0.241 & 0.207 \\
HV s/d & 0.057 & 0.771 \\
Staging (degree of fibrosis) & 0.126 & 0.516 \\
Grading (necroinflammatory response) & 0.110 & 0.570 \\
Blood loss (ml) & 0.236 & 0.257 \\
\hline
\end{tabular}


Data are presented by the correlation coefficient (r value).

PV; portal vein, HA; hepatic artery, HV; hepatic vein

ICG: indocyanine green, LHL15: liver uptake ratio at 15 minutes 
Table 4 Multiple linear regression stepwise method output using US Doppler data for correlations with portal pressure

\begin{tabular}{|c|c|c|c|c|c|c|c|c|}
\hline \multirow{2}{*}{\multicolumn{2}{|c|}{ Model }} & \multicolumn{2}{|c|}{ Unstandardized coefficients } & \multirow{2}{*}{$\frac{\text { Standardized coefficient }}{\beta}$} & \multirow[b]{2}{*}{ t value } & \multirow[b]{2}{*}{$P$ value } & \multicolumn{2}{|c|}{ 95\% confidence interval } \\
\hline & & $\beta$ & Standard error & & & & lower limit & upper limit \\
\hline \multirow[t]{6}{*}{1} & (Constant) & 18.303 & 2.025 & & 9.038 & .000 & 14.123 & 22.482 \\
\hline & PV max. & -.097 & .073 & -.275 & -1.323 & .198 & -.249 & .054 \\
\hline & PV min. & -.350 & .225 & -.421 & -1.558 & .132 & -.814 & .114 \\
\hline & PV ed. & .020 & .238 & .030 & .085 & .933 & -.471 & .512 \\
\hline & PV ps. & -.094 & .209 & -.152 & -.449 & .657 & -.526 & .338 \\
\hline & PV mean & .046 & .290 & .041 & .159 & .875 & -.553 & .646 \\
\hline \multirow[t]{5}{*}{2} & (Constant) & 18.324 & 1.969 & & 9.306 & .000 & 14.268 & 22.379 \\
\hline & PV max. & -.098 & .071 & -.277 & -1.372 & .182 & -.245 & .049 \\
\hline & PV min. & -.343 & .203 & -.412 & -1.688 & .104 & -.761 & .075 \\
\hline & PV ps. & -.083 & .156 & -.133 & -.529 & .601 & -.403 & .238 \\
\hline & PV mean & .051 & .279 & .046 & .184 & .856 & -.523 & .625 \\
\hline \multirow[t]{4}{*}{3} & (Constant) & 18.336 & 1.931 & & 9.496 & .000 & 14.367 & 22.306 \\
\hline & PV max. & -.100 & .069 & -.284 & -1.450 & .159 & -.242 & .042 \\
\hline & PV min. & -.320 & .158 & -.385 & -2.021 & .054 & -.646 & .005 \\
\hline & PV ps. & -.073 & .144 & -.117 & -.506 & .617 & -.369 & .223 \\
\hline \multirow[t]{3}{*}{4} & (Constant) & 18.235 & 1.894 & & 9.628 & .000 & 14.349 & 22.121 \\
\hline & PV max. & -.120 & .056 & -.340 & -2.156 & .040 & -.235 & -.006 \\
\hline & PV min. & -.364 & .131 & -.437 & -2.766 & .010 & -.633 & -.094 \\
\hline
\end{tabular}


Dependent variable is portal pressure (mmHg). PV; portal vein max; maximum, min; minimum, ed; endo-diastolic, ps; peak systolic

Dependent variable: Step 4 total. The standardized ß coefficient provides a measure of the contribution of each variable to the model.

The $t$ and $\mathrm{p}$ values provide an indication of the impact of each predictor variable. 